\title{
ANNEXE \\ Note sur la consolidation d'une couche d'argile d'épaisseur infinie surmontée d'une couche perméable sous l'action d'un chargement appliqué à la surface du sol \\ PAR F. SERRE \\ INGÉNIEUR-DOCTEUR, \\ INGÉNIEUR A L'OMNIUM FRANÇAIS D'ÉTUDES E'T DE RECHERCHES
}

Dans un récent article, paru dans le Génie Civil, nous avons étudié l'évolution, au cours du temps, du tassement d'une couche d'argile d'épaisseur infinie surmontée d'une couche de sable perméable de hauteur $h$, sous l'action d'un chargement instantané appliqué à la surface du sol.

La solution à laquelle nous sommes parvenus est basée sur des conditions aux limites critiquables et n'est absolument rigoureuse que si la couche de sable a une épaisseur nulle, le chargement étant alors appliqué directement à la surface de l'argile.

Nous avons admis, en effet, que les contraintes totales dans la couche de sable, qui sont à un instant donné entièrement encaissées par l'ossature solide, étaient indépendantes du temps et données par le tenseur de Boussinesg, correspondant à l'équilibre élastique d'un domaine semi indéfini, hypothèses qui, nous le verrons par la suite, sont incompatibles avec les conditions aux limites à la surface de la couche d'argile.

En fait, la seule facon rigoureuse de résoudre le problème consiste à étudier l'évolution, au cours du temps, des contraintes et des déformations aussi bien dans la couche de sable que dans celle d'argile.

Nous prendrons pour plan horizontal de référence $x o y$ la face supérieure de la couche d'argile d'épaisseur infinie, l'axe o $z$ vertical étant orienté positivement vers le bas.

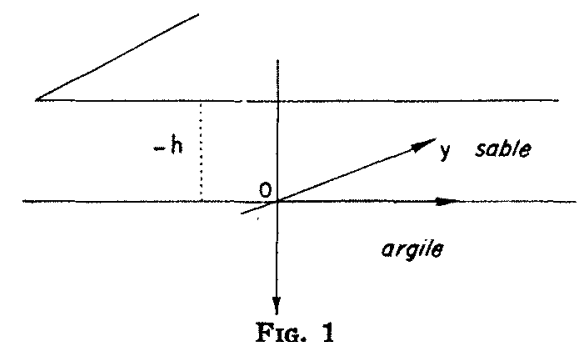

Nous désignerons respectivement par $\lambda$ et $\mu$, $\lambda_{1}$ et $u_{1}$, les coefficients de Lamé du sable et de l'argile.

\section{I. - Equations indéfinies}

Dans la couche d'argile, les équations indéfinies de la mécanique des sols s'écrivent :

$$
\left\{\begin{array}{c}
\left(\lambda_{1}+\mu_{1}\right) \operatorname{grad} \theta+\mu_{1} \Delta \overline{\mathbf{H}}=\text { grad } \rho \\
\theta=\operatorname{div} \overline{\mathbf{H}} \\
\frac{\partial \theta}{\partial t}-\frac{\mathbf{K}}{\bar{\omega}} \Delta \varphi=0
\end{array}\right.
$$

où $\mathbf{H}$ désigne le vecteur de déformation de composantes $(u, v, w), \theta$ la dilatation cubique, $\varphi$ la surpression hydrostatique de l'eau interstitielle, $K$ et $\bar{\omega}$, respectivement le coefficient de perméabilité et le poids spécifique de l'argile.

Les équations indéfinies précédentes sont applicables à la couche de sable perméable, mais elles se simplifient considérablement si on observe que le coefficient de perméabilité de celleci peut ètre considéré comme infini vis-à-vis $d u$ coefficient de perméabilité de l'argile. La dernière des équations (1) :

$$
\begin{gathered}
\frac{\bar{\omega}}{\kappa} \frac{\partial \theta}{\partial t}-\Delta \varphi=0 \\
\text { se réduit alors à } \Delta \varphi=0
\end{gathered}
$$

ce qui montre, compte tenu des conditions aux limites, que la surpression hydrostatique de l'eau interstitielle $\varphi$ est nulle dans la couche de sable dès l'instant initial.

Les contraintes et les déformations y satisfont donc, à tout instant, aux équations indéfinies de l'équilibre élastique (2) qui sont indépendantes du temps.

$$
(\lambda+\mu) \operatorname{grad}(\operatorname{div} \bar{H})+y . \Delta \bar{H}=0
$$

\section{II. - Conditions aux limites}

Un chargement, de révolution autour de $o z$, constitué par des forces de surface verticales, étant appliqué à l'instant $t=0$ à la surface du sol $z=-h$, il s'agit de trouver : 
a) Dans la couche d'argile $(z>o)$ une solution du système (1) régulière, quels que soient $x, y, z>0, t>o$

b) Dans la couche de sable $(-h<z<o)$ une solution du système (2) régulière, quels que soient $x, y, t>o$.

Ces solutions satisfaisant aux conditions aux limites suivantes :

$1^{\circ}$ A la surface du sol $(z=-h)$, les composantes du tenseur des contraintes $\left(\mathrm{N}_{3}, \mathrm{~T}_{1}, \mathrm{~T}_{2}\right)$, qui représentent l'action des forces extérieures appliquées à la surface du massif, doivent satisfaire aux conditions :

$$
\begin{array}{cc}
\mathrm{T}_{1}= & =0 \\
\mathrm{~T}_{2}=\quad & =0 \\
\mathrm{~N}_{3}=\lambda \theta+2 \mu \cdot(\partial \mathrm{w} / \partial z) & =-p(\rho)
\end{array}
$$

$p(\rho)$ étant la fonction de charge donnée supposée de révolution autour de $\mathrm{O} z\left(\rho=\sqrt{x^{2}}+\overline{y^{2}}\right)$;

$2^{\circ}$ Dans le plan de contact des deux couches $(z=0)$, il faut écrire l'égalité, pour chacune d'elles, du vecteur déformation, de composantes $(u, v, w)$, et du vecteur tension sur un élément plan horizontal, de composantes $\left(\mathrm{T}_{1}, \mathrm{~T}_{2}, \mathrm{~N}_{3}\right)$.

Enfin la surpression de l'eau interstitielle est nulle à un instant quelconque en tout point de la couche perméable de sable et en particulier à la surface de la couche d'argile. On en déduit pour $z=0$ la condition :

$$
\varphi(x, y, o, t)=0
$$

\section{III. - Condition initiale}

Cette condition concerne uniquement la couche d'argile puisque dans la couche de sable les équations indéfinies sont indépendantes du temps. La compressibilité de l'eau étant négligeable, la variation de volume instantanée d'une particule macroscopique située à l'intérieur de la couche d'argile ne peut provenir que de l'expulsion de l'eau interstitielle. Celle expulsion ne pouvant s'effectuer instantanément à l'instant initial, sauf en ce qui concerne la face supérieure de la couche d'argile, la déformation immédiate pour $t=0$, résultant de l'application des forces extérieures à la surface du massif, a lieu à volume constant.

On en déduit la condition initiale :

$$
0(x, y, z, o)=0
$$

\section{IV. - Détermination du tassement final}

Les équations indéfinies (1) et (2) admettent une solution indépendante du temps et satisfaisant aux conditions aux limites écrites plus haut $\operatorname{par}(\mathrm{I})$.

Dans la couche d'argile, cette solution satisfait aux équations (1) où on suppose que $\theta, \overline{\mathrm{H}}$ et o ne dépendent pas du temps; le système (1) s'écrit dans ces conditions :

$$
\left\{\begin{array}{l}
\Delta \varphi=0 \\
\theta=\operatorname{div} \overline{\mathrm{I}} \\
\left(\lambda_{1}+\mu_{1}\right) \operatorname{grad} \theta+\mu_{1} \Delta \bar{H}=\operatorname{grad} \varphi
\end{array}\right.
$$

La solution de l'équation de LAPLACE $\Delta \varphi=0$ régulière quel que soit $x, y$ et $z>0$, et qui satisfait à la condition $\varphi(x, y, o)=0$, est identiquement nulle.

Le système (1) se réduit donc à l'équation indéfinie de l'équilibre élastique :

$$
\left(\lambda_{1}+\mu_{1}\right) \operatorname{grad} \operatorname{div} \overline{\mathbf{H}}+\mu_{1} \Delta \overline{\mathbf{H}}=0
$$

La recherche d'une solution, indépendante du lemps, et satisfaisant aux conditions aux limites se ramène done finalement à un problème d'élasticité, relatif aux déformations de deux couches homogènes et isotropes de coefficients d'élasticité. respectifs, $\lambda$ et $\mu$ pour la couche de sable d'épaisseur $h, \lambda_{1}$ et $\mu_{1}$ pour la couche d'argile d'épaisseur infinie, sous l'action du chargement appliqué à la surface du massif.

Cette solution représente l'état vers lequel tend le système au bout d'un temps théoriquement infini; nous la désignerons par :

$$
\overline{\mathrm{H}}_{\infty}(x, y, z), \quad \theta_{\infty}(x, y, z), \quad \varphi_{\infty}(x, y, z)=0
$$

$\theta_{\infty}=\operatorname{div} \bar{H}_{\infty}$ est une intégrale de l'équation de LAPLACE $\Delta \theta=0$ que l'on obtient en prenant la divergence des deux membres de l'équation indéfinie de l'équilibre élastique.

Cette remarque nous conduit à rechercher pour $\theta_{c s}(x, y, z)$ une expression de la forme :

$$
\begin{array}{r}
a_{\infty}(x, y, z)=\int_{0}^{\infty}(\mathrm{A} \operatorname{ch} \sigma z+\mathrm{B} \operatorname{sh} \sigma z) \mathrm{J}_{0}(\sigma \rho) \sigma d \sigma \\
\left(\rho=\sqrt{x^{2}+y^{2}}\right)
\end{array}
$$

valable dans la couche de sable : $-h<Z<0$ et de la forme:

$$
\begin{aligned}
\theta_{\infty}(x, u, z)=\int_{0}^{+\infty} A_{1} e^{-\sigma z} J_{0}(\sigma \rho) \sigma d \sigma & \\
& \left(\rho=\sqrt{\left.x^{2}+y^{2}\right)}\right.
\end{aligned}
$$

valable dans la couche d'argile : $z>0$, 
où $A, B$ et $A_{1}$ sont des fonctions du paramètre $\sigma, J_{0}(u)$ désignant, suivant la notation habituelle, la fonction de Bessel d'ordre zéro, c'està-dire l'intégrale de l'équation différentielle :

$$
\frac{d^{2} f}{d u^{2}}+\frac{1}{u} \frac{d f}{d u}+f=0
$$

régulière quel que soit $u$ et satisfaisant à la condition $f(0)=1$.

Il est possible de disposer des fonctions arbitraires du paramètre $\sigma: A(\sigma), B(\sigma), A_{1}(\sigma)$ et de celles qui interviennent dans la détermination des composantes de la déformation $\overrightarrow{\mathrm{H}}_{\infty}$, de facon à satisfaire aux conditions aux limites. On est ainsi conduit à résoudre un système d'équations algébriques linéaires, la fonction de charge à la surface du massif $p$ ( $९$ ) étant mise préalablement sous la forme :

$$
p(\rho)=\int_{0}^{+\infty} \psi(\sigma) J_{0}(\sigma \rho) \sigma d \sigma
$$

La résolution de ce système se simplifie considérablement lorsque les coefficients de glissement des deux couches sont identiques $\mu_{1}=\mu$; dans la suite du problème, nous nous placerons délibérément dans cette hypothèse qui simplifie considérablement l'écriture.

Dans ces conditions $A(\sigma)$ est donné par l'expression :

$$
\begin{array}{r}
A(\sigma)=-\psi(\sigma) \frac{\left(\lambda_{1}+2 \mu\right) e^{\sigma n}}{\left(\lambda_{1}+2 \mu\right)(\lambda+\mu) e^{2 \sigma h}+\mu\left(\lambda_{1}-\lambda\right)} ; \\
A_{1}=\mathrm{A} \frac{\lambda+2 \mu}{\lambda_{1}+2 \mu}
\end{array}
$$

où $\psi(\sigma)$ est donné par la résolution de l'équation intégrale classique :

$$
p(\rho)=\int_{0}^{+\infty} \psi(\sigma) \mathrm{J}_{0}(\sigma \rho) \sigma d \sigma
$$

dont la solution s'obtient par la formule d'inversion :

$$
\psi(\sigma)=\int_{0}^{+\infty} p(\rho) J_{0}(\sigma \rho) \rho d \rho
$$

Plaçons-nous dans l'hypothèse d'une charge $P$ répartie uniformément sur un cercle de rayon $a$ :

$$
\begin{array}{ll}
p(\rho)=\frac{\mathrm{P}}{\pi a^{2}} & \text { pour } o<\rho<a \\
p(\rho)=0 & \text { pour } \circ>a
\end{array}
$$

il vient :

$$
\psi(\sigma)=\frac{\mathrm{P}}{\pi a^{2}} \int_{0}^{a} \mathrm{~J}_{0}(\sigma \rho) \rho d \rho=-\frac{\mathrm{P}}{\pi a \sigma} \mathrm{J}_{0}^{\prime}(\sigma a)
$$

où $J_{0}^{\prime}(u)$ désigne la dérivée de $J_{0}(u)$.

Dans l'hypothèse où nous nous placerons désormais d'une force concentrée, verticale $\mathrm{P}$ dirigée suivant l'axe de révolution oz, la fonction $\psi(\sigma)$ s'obtient en faisant $a=0$ dans la formule précédente, ce qui donne :

$$
\psi(\sigma)=\frac{P}{2 \pi}
$$

et :

$\mathrm{A}(\sigma)=-\frac{\mathrm{P}}{2 \pi} \frac{\left(\lambda_{1}+2 \mu\right) e^{\sigma h}}{\left(\lambda_{1}+2 \mu\right)(\lambda+\mu) e^{2 \sigma h}+\mu\left(\lambda_{1}-\lambda\right)}$

La dilatation cubique $0_{\infty}(x, y, z)$ et les composantes de la déformation $u_{\infty}, v_{\infty}, w_{\infty}$, s'en déduisent. On obtient en particulier pour valeur de $0_{\infty}$ dans la couche d'argile :

$$
\begin{aligned}
& \theta_{\infty}(x, y, z)= \\
& -\frac{\mathrm{P}}{2 \pi} \int_{0}^{+\infty} \frac{(\lambda+2 \mu) e^{\sigma h} \times e^{-\sigma z} \mathrm{~J}_{0}(\sigma \rho) \sigma d \sigma}{\left(\lambda_{1}+2 \mu\right)(\lambda+\mu) e^{2 \sigma h}+\mu\left(\lambda_{1}-\lambda\right)}
\end{aligned}
$$

et pour la composante verticale de la déformation à la surface de la couche de sable $(z=-h)$ :

$$
\begin{aligned}
& \mathrm{W}_{\infty}(x, y,-h)= \\
& \frac{\mathrm{P}(\lambda+2 \mu)}{4 \pi \mu \cdot} \int_{0}^{\infty} \frac{\left(\lambda_{1}+2 \mu\right) e^{2 \sigma h} \mathrm{~J}_{0}(\sigma \rho) d \sigma}{\left(\lambda_{1}+2 \mu\right)(\lambda+\mu) e^{2 \sigma h}+\mu\left(\lambda_{1}-\lambda\right)}
\end{aligned}
$$

qui donne le tassement final à la surface du massif.

A titre de vérification, on doit retrouver les lormules de Boussineso relatives à la déformation élastique d'un massif semi-indéfini homogène et isotrope, dans les deux cas suivants : $\lambda_{1}=\lambda$ les deux couches ont alors les même coefficients d'élasticité;

$h=0$ la couche de sable intermédiaire disparaît.

$$
1 " \lambda_{1}=\lambda \text {, il vient : }
$$

$$
\begin{aligned}
& \theta_{\infty}(x, y, z)=\frac{\mathrm{P}}{2 \pi(\lambda+\mu)} \int_{0}^{+\infty} e^{-\alpha(z+h)} \mathrm{J}_{0}(\sigma \rho) \sigma d \sigma \\
& =-\frac{\mathrm{P}(z+h)}{2 \pi(\lambda+\mu)\left\lceil\rho^{2}+(z+h)^{2}\right\rceil^{3 / 2}} \\
& \mathrm{~W}_{\infty}(x, y, h)=\frac{\mathrm{P}(\lambda+2 \mu)}{4 \pi \mu(\lambda+\mu .)} \frac{1}{\rho}
\end{aligned}
$$

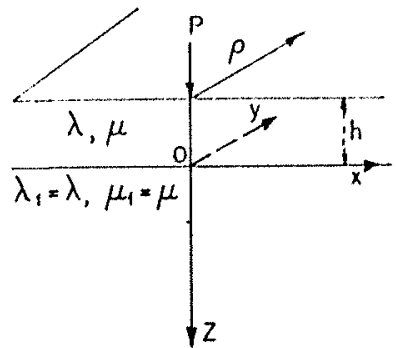

FIG. 2 
$2^{\circ} h=0:$

$$
\begin{array}{r}
\theta_{\infty}(x, y, z)=-\frac{\mathrm{P}}{2 \pi\left(\lambda_{1}+\mu\right)} \int_{0}^{\infty} e^{--\mathrm{u} z} \mathrm{~J}_{0}(\sigma \rho) \sigma d \sigma \\
=-\frac{\mathrm{P}}{2 \pi\left(\lambda_{1}+\mu\right)} \frac{z}{\left[\rho^{2}+z^{2}\right]^{3 / 2}} \\
\mathrm{~W}_{\infty}(x, y,-h)=\frac{\mathrm{P}\left(\lambda_{1}+2 \mu\right)}{4 \pi \mu\left(\lambda_{1}+\mu\right)} \frac{1}{\rho}
\end{array}
$$

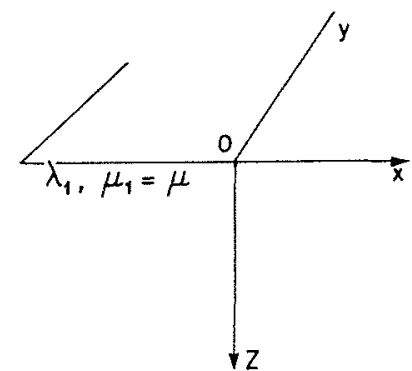

FIG. 3

\section{V. - Détermination de la solution complète}

C'est la solution qui satisfait à la fois aux conditions aux limites et à la condition initiale.

Désignons par $\overline{\mathbf{H}}_{1}(x, y, z, t), \varphi_{1}(x, y, z, t)$ la solution des équations indéfinies qui satisfait aux conditions aux limites rendues homogènes $\left(^{\star}\right)$ et à la condition initiale pour $t=0$.

$$
\theta_{1}(x, y, z, o)=\operatorname{div} \overline{\mathbf{H}}_{1}=-\theta_{\infty}(x, y, z)
$$

Les expressions :

$$
\left\{\begin{array}{l}
\overline{\mathbf{H}}(x, y, z, t)=\overline{\mathbf{H}}_{\infty}(x, y, z)+\overline{\mathbf{H}}_{1}(x, y, z, t) \\
\varphi(x, y, z, t)=\vartheta_{1}(x, y, z, t)
\end{array}\right.
$$

constituent la solution du problème.

En effet, ces combinaisons linéaires satisfont manif estement aux équations indéfinies, aux conditions aux limites et à la condition initiale :

$$
\theta(x, y, z, o)=\operatorname{div} \bar{H}(x, y, z, o)=0
$$

La fonction $\theta_{1}(x, y, z, t)$ est une intégrale de l'équation de Laplace $\Delta \theta=0$, dans la couche de sable, et de l'équation de Fourier :

$$
\left(\lambda_{1}+2 \mu_{1}\right) \frac{K}{\bar{\omega}} \Delta \theta-\frac{\partial \theta}{\partial t}=0
$$

dans la couche d'argile, car ces équations sont des conséquences des équations indéfinies (2) et (1).

${ }^{*}$ ( ) Ce sont les conditions écrites aux alinéas 1 et 2 du paragraphe II après suppression des seconds membres.
Cette remarque nous conduit à rechercher pour $0_{1}(x, y, z, t)$ une solution de la forme :

$$
\begin{aligned}
o_{1}(x, y, z, t)=\int_{0}^{+\infty} & \int_{0}^{+\infty} e^{-\left(\alpha^{2}+\sigma^{2}\right) \mathrm{C} t}\left[\mathrm{~A}_{1}(\alpha, \sigma) \cos \alpha z\right. \\
& \left.+\mathrm{A}_{2}(\alpha, \sigma) \sin \alpha z\right] J_{0}(\sigma, \sigma) \sigma d \sigma d \alpha
\end{aligned}
$$

valable dans la couche d'argile $z>0$ avec $\mathrm{C}=\left(\lambda_{1}+2 \mu_{1}\right) \mathrm{K} /$ w,

et de la forme :

$$
\begin{array}{r}
\theta_{1}(x, y, z, t)=\int_{0}^{+\infty} \int_{0}^{+\infty} e^{-\left(\alpha^{2}+\sigma^{2}\right) C \mathrm{t}}[\mathrm{A}(\alpha, \sigma) \operatorname{ch} \sigma z \\
+\mathrm{B}(\alpha, \sigma) \operatorname{sh} \sigma z\rceil \mathrm{J}_{0}(\sigma \rho) \sigma d \sigma d \alpha
\end{array}
$$

valable dans la couche de sable : $-h<z<0$.

Il est possible de disposer des fonctions arbitraires des paramètres $\alpha$ et $\sigma, A_{1}, A_{2}, A, B$, et de celles qui interviennent dans la détermination des composantes de $\overline{\mathrm{H}}_{1}$, de façon à satisfaire aux conditions aux limites rendues homogènes. On est ainsi amené à résoudre un système d'équations algébriques linéaires et homogènes dont le nombre est inférieur d'une unité à celui des inconnues.

Toutes celles-ci peuvent donc s'exprimer en fonction de l'une quelconque d'entre elles, $A_{1}$, par exemple.

La résolution de ce système se simplifie considérablement dans l'hypothèse, précédemment envisagee, où les coefficients de glissement des deux couches sont identiques, $\mu_{1}=\mu$.

Dans ces conditions, la relation entre $A_{1}$ et $\mathrm{A}_{2}$ prend la forme : :

$$
\begin{aligned}
\frac{\mathrm{A}_{2} \sigma^{2}+\mathrm{A}_{2} \gamma \sigma}{\alpha^{2}+\sigma^{2}}= & \frac{\lambda_{1}+2 \mu}{2 \mu(\lambda+2 \mu)} \\
& {\left[(\lambda+\mu) e^{2 \sigma h}+\mu\right] \mathrm{A}_{1} }
\end{aligned}
$$

la fonction $A_{1}(\alpha, \sigma)$ est alors déterminée par la condition initiale $\theta(x, y, z, o)=-\theta_{\infty}(x, y, z)$, ce qui conduit à la résolution de l'équation intégrale :

$$
\begin{aligned}
\frac{P}{2 \pi} & \frac{(\lambda+2 \mu) e^{\sigma h} e^{-\sigma \nu}}{\left.\left(\lambda_{1}+2 \mu\right) \lambda+\mu\right) e^{2 \sigma h}+\mu\left(\lambda_{1}-\lambda\right)} \\
= & \int_{0}^{+\infty}\left[A_{1}(\alpha, \sigma) \cos \alpha z+A_{2}(\alpha, \sigma) \sin \alpha z\right] d \alpha
\end{aligned}
$$

$A_{1}$ et $A_{2 .}$ étant liés par la relation (3).

$A_{1}$ et $A_{2}$ sont donnés par les formules :

$$
\begin{array}{r}
\mathrm{A}_{1}=\frac{\mathrm{P}}{2 \pi^{2}} \cdot \frac{(\lambda+2 \mu) e^{\sigma h}}{\left(\lambda_{1}+2 \mu\right)(\lambda+\mu) e^{2 \sigma h}+\mu\left(\lambda_{1}-\lambda\right)} \\
{\left[\frac{\sigma}{\alpha^{2}+\sigma^{2}}-\frac{\delta^{2} \sigma}{\alpha^{2}+\delta^{2} \sigma^{2}}\right]}
\end{array}
$$




$$
\begin{array}{r}
A_{s}=\frac{P}{2 \pi^{2}} \frac{(\lambda+2 \mu) e^{\sigma h}}{\left(\lambda_{1}+2 \mu\right)(\lambda+\mu) e^{2 \sigma h}+\mu\left(\lambda_{1}-\lambda\right)} \\
{\left[\frac{\alpha}{\alpha^{2}+\sigma^{2}}+\frac{\delta \alpha}{\alpha^{2}+\delta^{2} \sigma^{2}}\right]}
\end{array}
$$

où $\delta$ est une fonction du seul paramètre $\sigma$ donnée par l'expression :

$\hat{\partial}=\frac{\left(\lambda_{1}+2 \mu\right)(\lambda+\mu) e^{2 \sigma h}+\mu\left(\lambda_{1}+2 \mu\right)-2 \mu(\lambda+2 \mu)}{\left(\lambda_{1}+2 \mu\right)\left[(\lambda+\mu) e^{2 \sigma h}+\mu\right]}$
On en déduil l'expression en fonction du temps, du tassement (composante verticale de la déformation) d'un point quelconque de la face supérieure de la couche de sable, défini par sa distance $\rho$ à l'axe $o z$ :

$\tau\left(\hat{\imath}_{1} t\right)=\mathrm{W}(x, y,-h, t)=\mathrm{W}_{\infty}(x, y,-h)$

$-\int_{0}^{\infty} \int_{0}^{+\infty} e^{-\sigma\left(\alpha^{2}+\sigma^{2}\right) t} \times \frac{\lambda_{1}+2 \mu}{2 \mu} \mathrm{A}_{1}(\alpha, \sigma) e^{\sigma h} \mathrm{~J}_{0}(\sigma \rho) d \sigma d \alpha$

Cette intégrale double peut, par dérivation sous le signe somme, se ramener à une intégrale simple plus directement accessible au calcul.

On obtient, tous calculs faits :

$$
\tau(\rho, t)=\frac{\mathrm{P}(\lambda+2 \mu)}{8 \pi \mu} \int_{0}^{+\infty} \mathrm{R}(\sigma h) \mathrm{F}[\delta(\sigma), \sigma \sqrt{\mathrm{C} t}] J_{0}(\sigma \rho) d \sigma
$$

avec :

$$
\mathrm{R}(\sigma h)=\frac{\left(\lambda_{1}+2 \mu\right) e^{2 \sigma h}}{\left(\lambda_{1}+2 \mu\right)(\lambda+\mu) e^{2 \sigma h}+\psi \cdot\left(\lambda_{1}-\lambda\right)}
$$

et :

$$
\begin{aligned}
& \mathrm{F}[\delta(\sigma), \sigma \sqrt{\mathrm{C} t}]=1+\mathrm{G}(\sigma \sqrt{\mathrm{C} t}) \\
& \quad+\delta e^{-\left(1-\delta^{2}\right) \sigma^{2} \mathrm{C} t}[1-\mathrm{G}(\delta \sigma \sqrt{\mathrm{C} t})]
\end{aligned}
$$

$G(x)$ désignant, suivant la notation habituelle, la fonction de Gauss :

$$
\mathrm{G}(x)=\frac{2}{\sqrt{\pi}} \int_{0}^{x} e^{-u^{2}} d u
$$

le calcul numérique du tassement pour diverses valeurs des rapports $h / \rho, \sqrt{\mathrm{Ct}} / \rho$, s'effectue sous cette forme à l'aide des tables de Gauss et de BESSEL.

Pour $h=0$, on retrouve le résultat que nous avions déjà obtenu, os est alors indépendant de $\sigma$ et prend la valeur constante : $\delta=\lambda_{1} /\left(\lambda_{1}+2 \mu\right)$.

Le tassement initial est donné par l'expression :

$\tau(\rho, 0)=\frac{\mathrm{P}(\lambda+2 \mu)}{8 \pi \mu} \int_{0}^{+\infty} \mathrm{R}(\sigma h)(1+\delta) \mathrm{J}_{0}(\sigma \rho) d \sigma$

soit, compte lenu des valeurs de $\mathrm{R}(\sigma h)$ et $\delta$ données par (4) et (6) :

$$
\begin{aligned}
& \tau(\rho, o)=\mathrm{W}(x, y,-h, o)= \\
& \quad \frac{\mathrm{P}(\lambda+2 u)}{4 \pi \mu} \int_{0}^{+\infty} \frac{e^{2 \sigma h}}{(\lambda+u) e^{2 \sigma h}+\mu} \mathrm{J}_{0}(\sigma \rho) d \sigma
\end{aligned}
$$

il est indépendant du coefficient $\lambda_{1}$ de la couche d'argile. La comparaison avec le tassement final donné par l'expression :

$$
\begin{aligned}
\tau(\rho, \infty)= & W_{\infty}(x, y,-h)=\frac{\mathrm{P}(\lambda+2 \mu)}{4 \pi \mu} \\
& \int_{0}^{+\infty} \frac{\left(\lambda_{1}+2 \mu\right) e^{2 \mu h} J_{0}(\sigma 0) d \sigma}{\left(\lambda_{1}+2 \mu\right)(\lambda+\mu) e^{2}+\mu\left(\lambda_{1}-\lambda\right)}
\end{aligned}
$$

montre que le tassement initial peut s'obtenir en faisant $\lambda_{1}=\infty$ dans $\tau(0, \infty)$. Ce résultat est conforme au théorème sur la déformation immé. diate de M. Mandel.

Le degré de consolidation, c'est-à-dire le rapport $\gamma$ du tassement à l'instant $t$ au tassement final, est une fonction des coefficients sans dimensions $h / \rho$ et $\sqrt{\mathrm{Ct}} / \mathrm{\rho}$; il est donné par l'expression :

$\gamma=1-\frac{\int_{0}^{+\infty} \mathrm{R}\left(\alpha \frac{h}{\rho}\right) \Phi\left(\delta, \alpha \frac{\sqrt{C T}}{\rho}\right) \mathrm{J}_{0}(\alpha) d \alpha}{\int_{0}^{+\infty} \mathrm{R}\left(\alpha \frac{h}{\rho}\right) \mathrm{J}_{0}(\alpha) d \alpha}$

avec :

$$
\delta=1 \cdots \frac{2 \mu_{0}(\lambda+2 \mu)}{\left(\lambda_{1}+2 \mu\right)\left[(\lambda+\mu) e^{2 \alpha(h / p)}+\mu\right]}
$$

$\mathrm{R}\left(\alpha \frac{h}{\rho}\right)=\frac{\left(\lambda_{1}+2 \mu\right) e^{2 \alpha(h / p)}}{\left(\lambda_{1}+2 \mu\right)(\lambda+\mu) e^{2 \alpha(h / \mu)}+\mu\left(\lambda_{1}-\lambda\right)}$

$\Phi\left(\delta, \alpha \frac{\sqrt{\mathrm{C} t}}{\rho}\right)=1-\mathrm{G}\left(\alpha \frac{\sqrt{\mathrm{C} t}}{\rho}\right)$

$$
-\delta e^{-\left(1-\delta^{2}\right) \alpha^{2}\left(\mathrm{C} t / \rho^{2}\right)}\left[1-\mathrm{G}\left(\partial \alpha \frac{\sqrt{\mathrm{C} t}}{\rho}\right)\right]
$$


L'examen de ces formules montre l'influence, sur le degré de consolidation, de l'épaisseur $h$ de la couche perméable superficielle; si $h=0$, o garde une valeur constante indépendante de $\alpha$ :

$$
\delta=\frac{\lambda_{1}}{\lambda_{1}+2 \mu}<1
$$

et la fonction de $\alpha, \Phi(\delta, \alpha \sqrt{\mathrm{Ct}} / \rho)$, ne tend pas rapidement vers 0 pour les petites valeurs de $\sqrt{\mathrm{C} t} / \mathrm{\rho}$.

Le temps de consolidation $\mathrm{T}$, défini par la valeur $\mathrm{T}$ de $t$ pour laquelle le degré de consolidation $\gamma$ est égal à 0,99 , est alor's extrêmement long.

Sa durée est par contre considérablement réduite lorsque le rapport $h / q$ est grand. En effet, $\delta$ est alors une fonction de $\alpha$ qui tend très rapidement vers sa limite 1 , et $\Phi(\delta, \alpha \sqrt{\mathrm{Ct}} / \rho)$ est alors pratiquement nul quel que soit $\sqrt{\mathrm{C} t} / \mathrm{\rho}$. La consolidation au niveau de la fondation peut alors être quasi instantanée.

Ces résultats sont traduits par les courbes cicontre qui donnent $\gamma$ en fonction de $t$ pour diverses valeurs du rapport $h / \rho$.

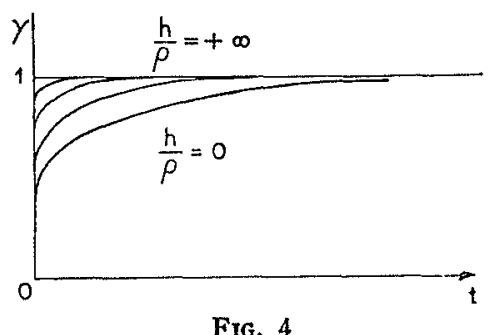

En conclusion, la fondation d'un ouvrage audessus d'une couche d'argile imperméable de grande épaisseur, peut pratiquement ne pas poser de problème de consolidation, lorsqu'elle est séparée de la fondation par une couche perméable d'épaisseur suffisante.

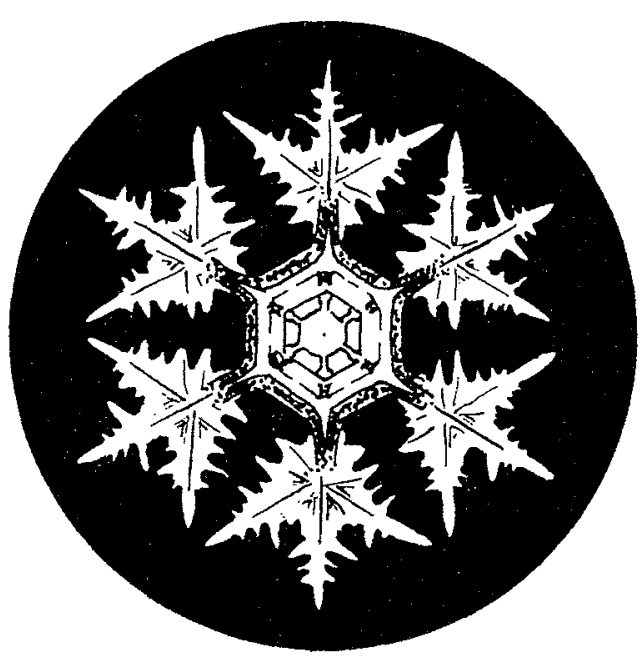

